\title{
Dependence of the gamma-ray emission from SN 1006 on the astronomical parameters
}

\author{
L. T. Ksenofontov ${ }^{1}$, E. G. Berezhko ${ }^{1}$, and H. J. Völk ${ }^{2}$
}

\author{
1 Yu.G.Shafer Institute of Cosmophysical Research and Aeronomy, 31 Lenin Av., 677980 Yakutsk, Russia \\ e-mail: [berezhko;ksenofon]@ikfia.ysn.ru \\ 2 Max-Planck-Institut für Kernphysik, Postfach 103980, 69029 Heidelberg, Germany \\ e-mail: Heinrich.Voelk@mpi-hd.mpg.de
}

Received 14 March 2005 / Accepted 21 July 2005

\section{ABSTRACT}

We use nonlinear kinetic theory to study the remnant dynamics and the particle acceleration as well as the properties of the nonthermal emission from the supernova remnant SN 1006. The known range of astronomical parameters is examined to determine whether it encompasses the existing synchrotron emission data. Given the present-day spatial extent and expansion rate of the object, it is shown that the hadronic gammaray flux is very sensitive to the ambient gas density $N_{\mathrm{H}}$ and that the existing HESS upper limit requires $N_{\mathrm{H}}<0.1 \mathrm{~cm}^{-3}$. The strength of the amplified magnetic field downstream of the shock is about $150 \mu \mathrm{G}$.

Key words. cosmic rays - ISM: supernova remnants

\section{Introduction}

Despite the widespread belief that the cosmic rays (CRs) below the so-called knee in the energy spectrum at a few $10^{15} \mathrm{eV}$ are accelerated in the shell-type supernova remnants (SNRs) of our Galaxy, this proposition still has only limited evidence. This is not due to an inadequate theoretical understanding. In fact, essentially all physical processes involved have either been clarified or, like the injection rates of ions and of electrons into the diffusive shock acceleration process, can be inferred from the observed radio and X-ray synchrotron spectra (for a recent overview, see Völk 2004). The limitation is rather the scarcity of SNR detections in $\mathrm{TeV} \gamma$-rays as an unequivocal signature of the existence of very high energy particles in these sources.

Of this $\mathrm{SN} 1006(\mathrm{G} 327.6+14.6)$ is the most prominent case. Observations of nonthermal X-rays (Koyama et al. 1995) suggest that at least CR electrons are accelerated in SN 1006 up to energies of about $100 \mathrm{TeV}$. Subsequent $\gamma$-ray observations with the CANGAROO telescopes (Tanimori et al. 1998, 2001) strengthened this conclusion. It was also shown (Berezhko et al. 2002) that nonlinear kinetic theory of CR acceleration is consistent with all observational data for a value $N_{\mathrm{H}}=$ $0.3 \mathrm{~cm}^{-3}$ of the ambient interstellar medium (ISM) density from the range $0.05 \leq N_{\mathrm{H}} \leq 0.3 \mathrm{~cm}^{-3}$ existing in the literature. However, SN 1006 could not be detected by the HESS experiment as a TeV source in a total of 18.2h (in 2003) and 6.3h (in 2004) livetime of ON source observations with SN 1006 in the field of view (Aharonian et al. 2005). The HESS upper limit is roughly one order of magnitude lower than the published CANGAROO flux.

In this paper we demonstrate that the HESS upper limit does not invalidate the theoretical picture (Berezhko et al. 2002) on which the previous calculation of the $\gamma$-ray emission spectrum has been based. As we shall show, it is rather the value of the external astronomical parameter $N_{\mathrm{H}}$ that strongly influences the hadronic $\gamma$-ray flux. The Inverse Compton (IC) $\gamma$-ray flux, on the other hand, depends only weakly on $N_{\mathrm{H}}$. We use this theory to describe all the relevant properties of SN 1006 and re-examine the most relevant set of physical and astronomical parameters, mainly $N_{\mathrm{H}}$, the total explosion energy and, to a minor extent, the interior magnetic field strength as well as the distance to the object, given the HESS upper limit.

\section{SNR parameters}

Since SN 1006 is a type Ia supernova it presumably expands into a uniform ISM, ejecting roughly a Chandrasekhar mass $M_{\mathrm{ej}}=1.4 M_{\odot}$, which is characterized by the initial velocity distribution $\mathrm{d} M_{\mathrm{ej}} / \mathrm{d} v \propto v^{2-k}$ with $k=7$. We note that, since SN 1006 is already in the Sedov phase, its properties are not sensitive to the parameter values $M_{\mathrm{ej}}$ and $k$ of the ejecta. The ISM gas density $\rho_{0}=1.4 m_{\mathrm{p}} N_{\mathrm{H}}$, which is usually characterized by the hydrogen number density $N_{\mathrm{H}}$, is an important parameter that strongly influences the expected SNR 
dynamics and nonthermal emission. However, the value of $N_{\mathrm{H}}$ is poorly constrained for SN 1006 from the thermal emission observations. They yield a wide range of possible values from $N_{\mathrm{H}}=0.05-0.1 \mathrm{~cm}^{-3}$ (Dwarkadas \& Chevalier 1998) to $N_{\mathrm{H}} \approx 0.3 \mathrm{~cm}^{-3}$ (Dubner et al. 2002).

As in our early study (Berezhko et al. 2002) we apply here nonlinear kinetic theory of CR acceleration in SNRs (Berezhko et al. 1996; Berezhko \& Völk 1997) to find the optimum set of physical parameters of SN 1006, mainly the value of $N_{\mathrm{H}}$, which give a consistent description of the observed overall dynamics and of the nonthermal emission. As described in detail in these papers, that theory includes all the important physical factors which influence CR acceleration and SNR dynamics: shock modification by the CR backreaction, Alfvén wave damping within the shock transition, selfconsistent determination of the CR spectrum, and spatial distribution in each evolutionary phase. In addition it includes synchrotron losses of CR electrons and a determination of all nonthermal emission processes produced in SNRs by accelerated CRs (e.g. Berezhko et al. 2002, 2003a). It was demonstrated for the cases of SN 1006 (Berezhko et al. 2002) and Cassiopeia A (Berezhko et al. 2003a) that the values of these key parameters (proton injection rate, electron to proton ratio and interior magnetic field strength) which cannot be predicted theoretically with the required accuracy, can be determined from a fit of the observed synchrotron emission data. It is important here that the parameter values for these SNRs, determined in this way, were very well confirmed by the Chandra measurements of the fine structure of the nonthermal X-ray emission (Berezhko et al. 2003b; Berezhko \& Völk 2004).

We take as the most reliable estimate for the distance $d=2.2 \mathrm{kpc}$ to SN 1006 (Winkler et al. 2003). This distance is roughly $20 \%$ larger than the value $d=1.8 \mathrm{kpc}$ adopted in Berezhko et al. (2002).

\subsection{Ambient gas density and total explosion energy}

The SNR dynamics are illustrated in Fig. 1. In Fig. 1a we present the time dependence of the shock radius $R_{\mathrm{S}}$ and of the shock speed $V_{\mathrm{s}}$, calculated for the optimum upstream magnetic field value $B_{0}=30 \mu \mathrm{G}$ (see below), and for the expected density range of the ambient ISM. The value of the explosion energy was taken to fit the observed size and speed at the current epoch $t \approx 10^{3}$ yr. Since SN 1006 has already evolved into the Sedov phase, the explosion energy scales as $E_{\mathrm{sn}} \propto N_{\mathrm{H}}$. The calculations correspond to $E_{\mathrm{sn}} /\left(10^{51} \mathrm{erg}\right)=1.9,3.8$ and 11.4 , for $N_{\mathrm{H}} /\left(1 \mathrm{~cm}^{-3}\right)=0.05,0.1$ and 0.3 , respectively.

The scaling $E_{\mathrm{sn}} \propto N_{\mathrm{H}}$ is intuitively plausible, since for lower external density both the expansion velocity as well as the radius $R_{\mathrm{S}}(t)$ of the SNR are larger for a given explosion energy $E_{\mathrm{sn}}$. Asymptotically - and approximately, since there exists no strictly self-similar solution with particle acceleration - in the Sedov phase, we have $R_{\mathrm{S}}(t) \propto\left(E_{\mathrm{Sn}} / N_{\mathrm{H}}\right)^{1 / 5} t^{2 / 5}$. Therefore the ratio $E_{\mathrm{sn}} / N_{\mathrm{H}}$ is fixed for known $t, R_{\mathrm{s}}(t)$ and $V_{\mathrm{s}}(t)=\mathrm{d} R_{\mathrm{s}}(t) / \mathrm{d} t$.

The calculations shown in Fig. 1a demonstrate that with the dependence of $E_{\mathrm{sn}}$ on $N_{\mathrm{H}}$ the SNR dynamics are indeed



Fig. 1. Shock radius $R_{\mathrm{s}}$ and shock speed $V_{\mathrm{s}}$ as a function of time for the upstream magnetic field $B_{0}=30 \mu \mathrm{G}$ and different ISM number densities $N_{\mathrm{H}}(\mathbf{a})$, and for ISM number density $N_{\mathrm{H}}=0.1 \mathrm{~cm}^{-3}$ and different values of the upstream magnetic field $B_{0}(\mathbf{b})$. The observed size and speed of the shock (Moffett et al. 1993) are shown as well.

compatible with the measured values of $R_{\mathrm{S}}(t)$ and $V_{\mathrm{s}}(t)$ for different ISM densities. We note that the small differences in $V_{\mathrm{S}}(t)$ for different $N_{\mathrm{H}}$ in Fig. 1a during the Sedov phase $t>400 \mathrm{yr}$ are the result of the shock modification, which depends on $N_{\mathrm{H}}$ (see Fig. 2).

In addition, Fig. $1 \mathrm{~b}$ demonstrates the expected weak dependence of the dynamics on the magnetic field strength $B_{0}$. For a higher magnetic field $B_{0}$ the shock compression ratio $\sigma$ is lower (compare Fig. 2 with Fig. 1b in Berezhko et al. 2002). Therefore the postshock internal pressure $P_{2} \approx \rho_{0} V_{\mathrm{s}}^{2}(1-1 / \sigma)$ is higher for lower $B_{0}$. Since in the Sedov phase the thermal energy is the dominant form of energy, the total energy is $E_{\mathrm{sn}} \propto R_{\mathrm{s}}^{3} P_{2}$. Since $E_{\mathrm{sn}}$ is a constant the shock size $R_{\mathrm{s}}$ and therefore the shock speed $V_{\mathrm{s}}$ are somewhat larger for larger magnetic field $B_{0}$, in agreement with Fig. 1 . 


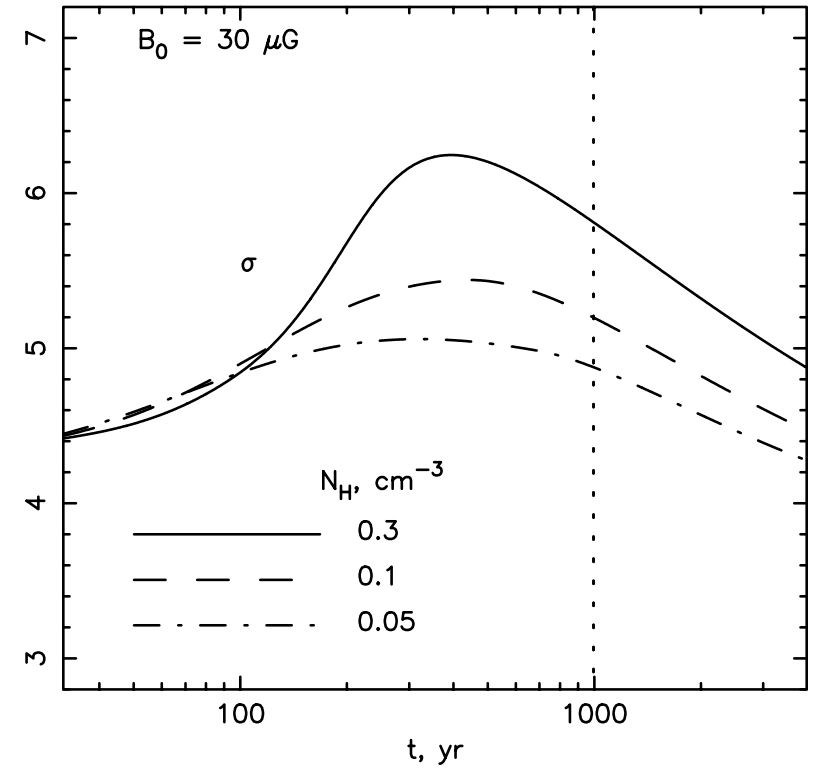

Fig. 2. The overall shock compression ratio $\sigma$ as a function of time, for three different ISM densities $N_{\mathrm{H}}=0.05,0.1$, and $0.3 \mathrm{~cm}^{-3}$, and upstream field $B_{0}=30 \mu \mathrm{G}$.

\subsection{Amplified B-field}

In our model the interior (downstream) magnetic field $B_{\mathrm{d}}$ is connected to the outer (upstream) magnetic field $B_{0}$ by the simple relation $B_{\mathrm{d}}=\sigma B_{0}$, where $\sigma$ is the overall shock compression ratio (Berezhko et al. 2002). It is also assumed that already the upstream field $B_{0}$ is significantly amplified as a consequence of CR streaming, and that it therefore substantially exceeds the existing ISM value (Lucek \& Bell 2000; Bell \& Lucek 2001; Bell 2004).

The shock compression ratio depends on the ambient gas density, as shown in Fig. 2. The behavior of the shock compression ratio $\sigma$ is determined by the values of two parameters: the proton injection rate $\eta$ and the Alfvénic shock Mach number $M_{\mathrm{a}}=V_{\mathrm{s}} / c_{\mathrm{a}}$, where $c_{\mathrm{a}}=B_{0} / \sqrt{4 \pi \rho_{0}}$ is the Alfvén speed. The value of the injection rate $\eta$ influences the critical shock speed $V_{\mathrm{s}}^{*} \propto \eta$ (Berezhko \& Ellison 1999) which separates the initial SNR evolutionary period, when $V_{\mathrm{s}}>V_{\mathrm{s}}^{*}$ and therefore the shock is almost unmodified, from the subsequent period $V_{\mathrm{s}}<V_{\mathrm{s}}^{*}$ of the modified shock. In our case $\eta=2 \times 10^{-4}$ and $V_{\mathrm{s}}^{*} \approx 10^{3} \mathrm{~km} \mathrm{~s}^{-1}$. During the period $V_{\mathrm{s}}<V_{\mathrm{s}}^{*}$ the shock compression ratio goes as $\sigma \approx 1.5 M_{\mathrm{a}}^{3 / 8}$ (Berezhko \& Ellison 1999). Therefore a decrease of the ISM density $N_{\mathrm{H}}$ leads to a decrease of the shock compression ratio $\sigma \propto V_{\mathrm{s}}^{3 / 8} N_{\mathrm{H}}^{3 / 16}$, as seen in Fig. 2 for $t>400 \mathrm{yr}$ (see also Fig. 1a). Thus, for the case $B_{0}=30 \mu \mathrm{G}$ used in Fig. 1a, the downstream magnetic field strengths are $B_{\mathrm{d}}=149,156$ and $173 \mu \mathrm{G}$ for $N_{\mathrm{H}}=0.05,0.1$ and $0.3 \mathrm{~cm}^{-3}$, respectively.

\subsubsection{Spatially integrated synchrotron spectrum}

For the global determination of the effective downstream field $B_{\mathrm{d}}$ we compare the theoretical synchrotron spectrum, calculated for three different values of $B_{0}$, with the observed spatially integrated spectrum (see Fig. 3).

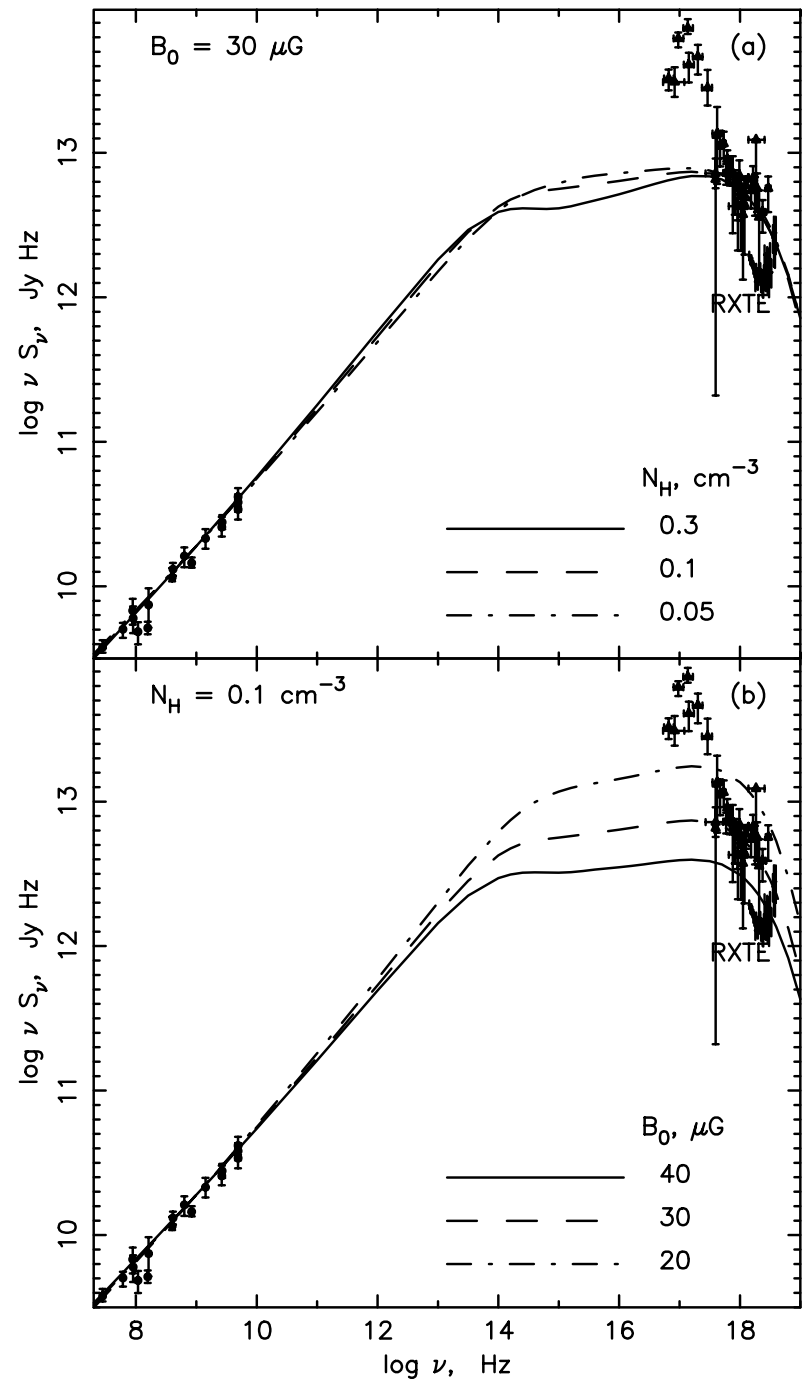

Fig. 3. Synchrotron emission flux as a function of frequency for the same cases as in Fig. 1. The observed X-ray (Hamilton et al. 1986; Allen et al. 1999) and radio emission (Reynolds 1996) fluxes are shown.

Electrons with a power-law energy spectrum $N_{\mathrm{e}}(\epsilon)=A_{\mathrm{e}} \epsilon^{-\gamma}$ produce a synchrotron flux $S_{v}=A v^{-\alpha}$ with spectral index $\alpha=(\gamma-1) / 2$ and amplitude $A \propto A_{\mathrm{e}} B_{\mathrm{d}}^{(\gamma+1) / 2} / d^{2}$. In the testparticle limit, $\gamma=2$ and therefore $\alpha=0.5$. Values $\alpha>0.5$, as observed in young SNRs, require a curved electron spectrum (hardening to higher energies) as predicted by nonlinear shock acceleration models. The synchrotron emission at frequency $v$ is mainly produced by electrons of energy $\epsilon=$ $5 \sqrt{[v /(1 \mathrm{GHz})]\left[(10 \mu \mathrm{G}) / B_{\mathrm{d}}\right]} \mathrm{GeV}$. Since the typical particle spectrum produced by a CR modified shock (Berezhko et al. 1996) is characterized by $\gamma>2$ at $\epsilon<1 \mathrm{GeV}$ and $\gamma<2$ at $\epsilon>10 \mathrm{GeV}$ it follows that in order to have $\alpha=0.57$, observed for SN 1006, numerical iteration shows that one needs efficient $\mathrm{CR}$ acceleration with a proton injection rate $\eta=2 \times 10^{-4}$, which leads to the required shock modification, and also to a high interior magnetic field $B_{\mathrm{d}} \geq 120 \mu \mathrm{G}$ (Berezhko et al. 2002). X-ray synchrotron spectral measurements are required to find the optimum value of the magnetic field strength $B_{\mathrm{d}}$ (Berezhko et al. 2002, 2003a): for a given fit of the synchrotron spectrum in the 
radio range the $\mathrm{X}$-ray synchrotron amplitude is very sensitive to $B_{\mathrm{d}}$ (see Fig. $3 \mathrm{~b}$ ).

An increase of the ISM number density $N_{\mathrm{H}}$ leads to an increase of the total number of low energy CRs. Since the radio emission is produced by low energy electrons and since for known SNR angular size and expansion rate the shock radius $R_{\mathrm{s}}$ and shock speed $V_{\mathrm{s}}$ are proportional to distance $d$, we have $A_{\mathrm{e}} \propto \eta V_{\mathrm{s}} R_{\mathrm{s}}^{3} K_{\mathrm{ep}} N_{\mathrm{H}} \propto \eta d^{4} K_{\mathrm{ep}} N_{\mathrm{H}}$. Therefore the amplitude of the synchrotron flux $A \propto \eta K_{\mathrm{ep}} N_{\mathrm{H}} d^{2} B_{\mathrm{d}}^{(\gamma+1) / 2}$. Since $A$ is fixed by the experiment, the electron to proton ratio changes like $K_{\text {ep }} \propto N_{\mathrm{H}}^{-1}$ for a given injection rate $\eta$ and interior magnetic field $B_{\mathrm{d}}$. Therefore we have $K_{\mathrm{ep}}=2.6 \times 10^{-3}, 1.1 \times 10^{-3}$ and $4.2 \times 10^{-4}$ for $N_{\mathrm{H}}=0.05,0.1$ and $0.3 \mathrm{~cm}^{-3}$ respectively. This includes the renormalization factor 0.2 for the overall ion intensity. For a given $N_{\mathrm{H}}$ we have $K_{\mathrm{ep}} \propto d^{-2} B_{\mathrm{d}}^{-(\gamma+1) / 2}$. Given that the Galactic CRs have a value $K_{\text {ep }} \simeq 10^{-2}$, the small value of $N_{\mathrm{H}}$ suggested by the HESS experiment makes SN $1006-$ at the present stage of evolution - more closely resemble an average source of the Galactic CRs.

As a result the synchrotron spectrum is almost insensitive to $N_{\mathrm{H}}$. The weak dependence of $S_{v}(v)$ on $N_{\mathrm{H}}$ is caused by the different downstream magnetic field $B_{\mathrm{d}}=\sigma B_{0}$ at the same $B_{0}$, because the compression ratio $\sigma$ depends on $N_{\mathrm{H}}$. For larger $N_{\mathrm{H}}$ the shock is more strongly modified. This leads to a harder CR spectrum at high energies $\epsilon \gg m_{\mathrm{p}} c^{2}$ and therefore the spectrum $S_{v}(v)$ at $v=10^{15}-10^{18} \mathrm{~Hz}$ becomes harder for larger $N_{\mathrm{H}}$, as can be seen in Fig. 3a.

In addition, an increase of the magnetic field $B_{\mathrm{d}}$ leads to a decrease of the electron energy $\epsilon_{\mathrm{l}} \propto t^{-1} B_{\mathrm{d}}^{-2}$ (Berezhko et al. 2002), where the electron spectrum undergoes a brake: for $\epsilon>\epsilon_{1}$ synchrotron losses in the downstream region are significant and lead to a steep overall (spatially integrated) electron spectrum $N_{\mathrm{e}} \propto \epsilon^{-\gamma-1}$, where $\gamma$ is the power law index of the CR spectrum at the shock front. Therefore the synchrotron spectrum has a break at the corresponding frequency $v_{1} \propto \epsilon_{1}^{2} B_{\mathrm{d}} \propto B_{\mathrm{d}}^{-3}$, which decreases with increasing $B_{\mathrm{d}}$, as one can see in Fig. 3 .

An increase of the magnetic field requires a reduction of the amplitude of the electron momentum distribution since the radio electrons are not subject to synchrotron cooling at the present epoch. This leads to a decreased electron/proton ratio $K_{\text {ep }} \propto B_{0}^{-\alpha-1}$ in the energy range where radiative cooling is unimportant. Most importantly however, the cooling region of the synchrotron emission spectrum $v>v_{1}$ will be lowered in amplitude by this decreasing electron/proton ratio. Comparison with the experimental X-ray data shows that the optimum magnetic field value is about $B_{0}=30 \mu \mathrm{G}$, and corresponds to a downstream field $B_{\mathrm{d}} \approx 150 \mu \mathrm{G}$. This is somewhat larger than the $B_{\mathrm{d}} \approx 120 \mu \mathrm{G}$ quite conservatively estimated earlier (Berezhko et al. 2002, 2003b), and is in agreement with the field amplification that is implied by the filamentary structures in hard X-rays, observed with Chandra (Bamba et al. 2003; Völk et al. 2005) (see also below).

As it was already noted (Berezhko et al. 2002), it is only the highest energy part of the X-ray spectrum corresponding to $v>10^{18} \mathrm{~Hz}$ that is of nonthermal origin. At lower frequencies $v \ll 10^{18} \mathrm{~Hz}$ the spectrum is dominated by thermal emission.

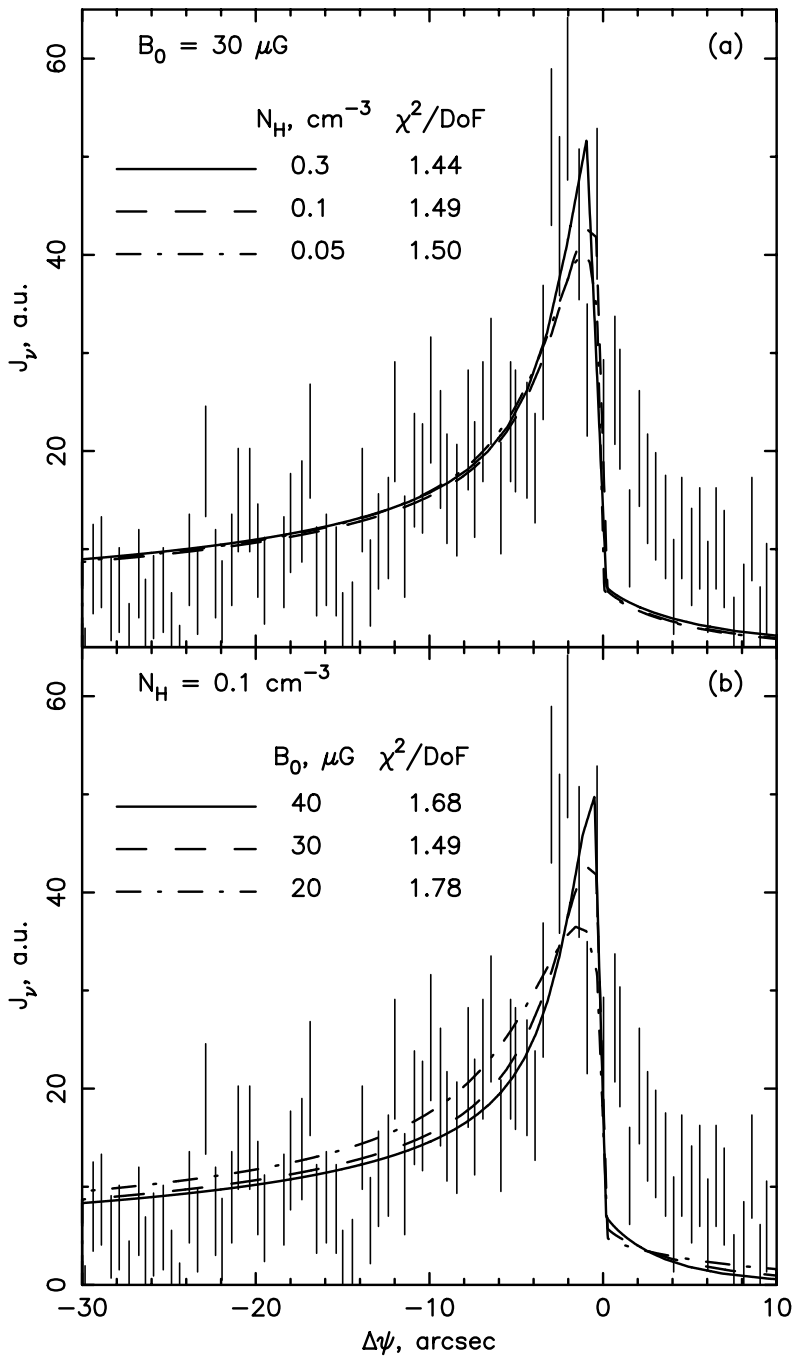

Fig. 4. Projected radial dependence of the non-thermal X-ray brightness in the energy range 2 to $10 \mathrm{keV}$, calculated for the same cases as in Fig. 1 together with the Chandra data corresponding to the sharpest profile in Bamba et al. (2003).

Therefore the theoretical spectrum for $v \ll 10^{18} \mathrm{~Hz}$ should be substantially below the experimental values, as it is in Fig. 3.

\subsubsection{Overall synchrotron morphology}

We now compare this globally determined magnetic field value $B_{0}=30 \mu \mathrm{G}$ with the local Chandra results by plotting the full numerical solutions for the overall SNR morphology, as it results from selfconsistent time-dependent kinetic theory, jointly with the sharpest of the experimental brightness profiles obtained by the Chandra observers (Bamba et al. 2003) in Fig. 4a, where the values of $\chi^{2} /$ d.o.f. characterizing the quality of fit are also shown. Since this profile obviously lies on a high superimposed background, we have subtracted from the data the constant brightness value which corresponds to the far upstream region. Note that the initial Chandra data were presented as a function of the angular radial distance $\psi$ (Bamba et al. 2003). The transformation to the linear projection distance $\rho=\psi d$ has been achieved by assuming a distance of $d=2.2 \mathrm{kpc}$ to 


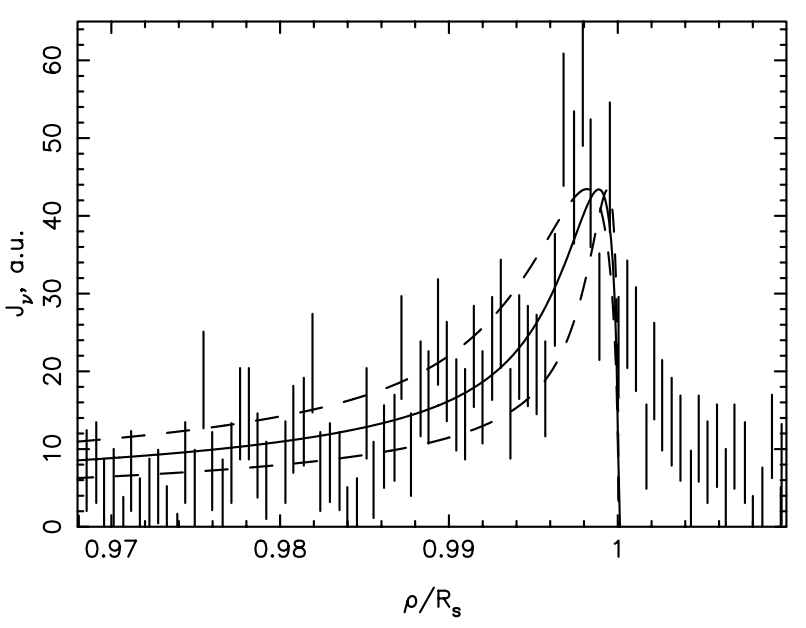

Fig. 5. The same Chandra $2-10 \mathrm{keV}$ radial continuum brightness profile as in Fig. 4, fitted to the projection of the exponential emissivity profile described by the model of Eq. (2). The fit has a $\chi^{2} /$ d.o.f. $=1.2$. The dashed lines indicate the $1 \sigma$ deviation.

SN 1006 (see above). We use in Figs. 4, $5 \Delta \psi$ instead of $\psi$, taking the initial point $\Delta \psi=0$ at the theoretically predicted shock position. The agreement of global morphology and local profiles is reasonable for all considered ISM densities.

Since the absolute values of the experimental brightness profiles and the actual shock position are not known their values were used as adjusting parameters in the fitting procedure.

Note that the radial profiles shown in Fig. 4a depend on $N_{\mathrm{H}}$ as a result of the dependence of the downstream magnetic field $B_{\mathrm{d}}=\sigma B_{0}$ on $N_{\mathrm{H}}$ : for higher $N_{\mathrm{H}}$ the compression ratio $\sigma$ is larger (see Fig. 2) and leads to a higher $B_{\mathrm{d}}$ and therefore to a thinner profile. In addition, the radial profile depends on the downstream plasma speed $u_{2}=V_{\mathrm{s}} / \sigma$ (Berezhko \& Völk 2004). The lower value of $u_{2}$, that is associated with a larger $N_{\mathrm{H}}$, makes the profile thinner.

As can be seen from Fig. $4 \mathrm{~b}$, the magnetic field value $B_{0}=$ $20 \mu \mathrm{G}$ slightly overestimates the profile width. Together with the spectral fit (Fig. 3b) we conclude that $B_{0}=30 \mu \mathrm{G}\left(B_{\mathrm{d}} \approx\right.$ $150 \mu \mathrm{G})$ is a realistic estimate.

\subsubsection{Local fit of the synchrotron profile}

$\mathrm{X}$-ray brightness profiles can also be used for an independent determination of the interior magnetic field value that does not require the nonlinear theory (Fig. 5).

It has been shown (Berezhko et al. 2003b; Berezhko \& Völk 2004) that in the case of strong synchrotron losses, which in fact occur for the electrons responsible for the X-ray synchrotron radiation, the downstream diffusion length over a synchrotron loss time mainly determines the three-dimensional thickness of the X-ray emission profile. The effective magnetic field can then be expressed in terms of the width $l_{2}$ of the radial synchrotron emissivity profile $q_{v}(r)$ according to the simple relation

$B_{\mathrm{d}}=\left(\frac{3 m_{\mathrm{e}}^{2} c^{4}}{4 e r_{0}^{2} l_{2}^{2}}\right)^{1 / 3} \approx 0.5\left(\frac{10^{16} \mathrm{~cm}}{l_{2}}\right)^{2 / 3} \mathrm{mG}$, where $m_{\mathrm{e}}, e$ and $r_{0}$ denote the electron mass, charge, and classical radius, respectively. The actual value of $l_{2}$ is determined from the observed width of the projected radial brightness profile

$J_{v}(\rho)=\int_{-\infty}^{\infty} q_{v}\left(r=\sqrt{\rho^{2}+x^{2}}\right) \mathrm{d} x$,

where $\rho$ is the projected distance from the remnant center and the integration is performed along the line of sight.

Since the synchrotron emissivity can be represented in the form $q_{v}(r)=q_{2} \exp \left[\left(r-R_{\mathrm{s}}\right) / l_{2}\right]$ (Berezhko et al. 2002), the projected profile is expressed in the analytical form

$J_{v} \approx q_{2} \frac{R_{\mathrm{s}} \sqrt{2 \pi R_{\mathrm{s}} l_{2}}}{\rho} \exp \left(-t^{2}\right) \operatorname{erfi}(t)$,

where $t=R_{\mathrm{S}} \sqrt{R_{\mathrm{S}}^{2}-\rho^{2}} /\left(\rho \sqrt{2 R_{\mathrm{S}} l_{2}}\right)$, and where $\operatorname{erfi}(x)$ is an imaginary error function. Note that the profile $J_{v}(\rho)$ can be expressed in terms of elementary functions (Berezhko \& Völk 2004), if we use the expansion of $\operatorname{erfi}(t)$ near the point $t=0$.

As shown in Fig. 5, the experimental Chandra values are quite well fitted by the analytical profile, described by Eq. (2), with an emissivity width $l_{2}=13.7(+8.2-6.2) \times 10^{-3} \mathrm{pc}$, that results in the effective magnetic field $B_{\mathrm{d}}=191(+82-62) \mu \mathrm{G}$. This range of downstream $B$-field strengths includes the values following for the three densities considered in Fig. 1a on the basis of an upstream field of $B_{0}=30 \mu \mathrm{G}$ which we shall use for the following.

Taking into account these results together with the consideration presented in Fig. 3 we conclude that $B_{0}=30 \mu \mathrm{G}$ and $B_{\mathrm{d}} \approx 150 \mu \mathrm{G}$ are the most appropriate values consistent with the existing data.

\section{Nonthermal energy density}

As we have seen, $E_{\mathrm{sn}} \propto N_{\mathrm{H}}$ in the Sedov phase, for given SNR size and expansion rate. Because these parameters are independent of time, it is necessary to choose this proportionality generally. Figure 1 demonstrates that this approach is roughly consistent for all phases. The resulting values for $E_{\mathrm{c}}$ in Fig. 6 are also calculated on this assumption.

In Fig. 6 we present the relative CR energy content $E_{\mathrm{c}} / E_{\mathrm{sn}}$ as a function of time, calculated for $B_{0}=30 \mu \mathrm{G}$ and three different ISM number densities $N_{\mathrm{H}}$. It increases with time, reaches a peak value at the epoch $t \simeq 3000 \mathrm{yr}$ and then slowly decreases due to an increasing dominance of adiabatic cooling. In the early free expansion phase $t \ll 100 \mathrm{yr}$, when nonlinear shock modification is not essential (see Fig. 2), the shock produces a power law CR spectrum $f \propto N_{\text {inj }} p_{\text {inj }} p^{-4}$, where $N_{\text {inj }}=\eta N_{\mathrm{H}}$ is the number of gas particles injected into the acceleration from each unit of volume intersecting the shock front and $p_{\text {inj }} \propto V_{\mathrm{s}}$ is their momentum. Since the strong unmodified shock always produces a CR spectrum of the same shape, the CR energy content $E_{\mathrm{c}} \propto N_{\mathrm{c}}$ at this stage grows proportionally to the total number $N_{\mathrm{c}}$ of accelerated CRs if we neglect weakly time dependent factor $\ln \left(p_{\max } / m_{\mathrm{p}} c\right)$, where $p_{\max }$ is maximum CR momentum. Assuming spherical symmetry for the moment, and starting from the obvious relation

$\mathrm{d} N_{\mathrm{c}} \propto N_{\mathrm{H}} R_{\mathrm{s}}^{2} V_{\mathrm{s}}^{2} \mathrm{~d} t$ 


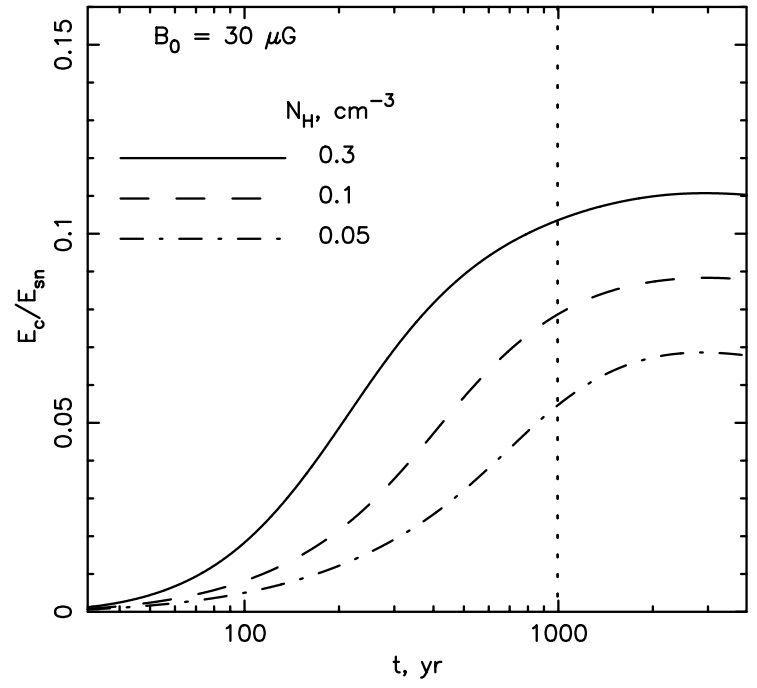

Fig. 6. Total energy $E_{\mathrm{c}} / E_{\mathrm{sn}}$ of accelerated particles in the SNR, normalized to the total hydrodynamic explosion energy $E_{\mathrm{sn}}$, as a function of time for the different ISM densities $N_{\mathrm{H}}$ shown in Figs. 1-5, assuming spherical symmetry. $E_{\mathrm{c}}$ is renormalized by a factor of 0.2 (cf. Völk et al. 2003). The upstream magnetic field strength is $B_{0}=30 \mu \mathrm{G}$.

and taking into account the shock expansion law (e.g. Chevalier 1982)

$R_{\mathrm{S}} \propto\left(E_{\mathrm{sn}}^{2} / N_{\mathrm{H}}\right)^{1 / 7} t^{4 / 7}$

we have

$E_{\mathrm{c}} \propto E_{\mathrm{sn}}^{8 / 7} N_{\mathrm{H}}^{3 / 7} t^{9 / 7}$

Then we have a residual density dependence at this early phase $E_{\mathrm{c}} / E_{\mathrm{sn}} \propto N_{\mathrm{H}}^{4 / 7}$ if we use $E_{\mathrm{sn}} \propto N_{\mathrm{H}}$.

In the transition to the Sedov phase (which happens earlier for larger $N_{\mathrm{H}}$ ), $E_{\mathrm{c}} / E_{\mathrm{sn}}$ slowly reaches a maximum as a function of time which is roughly independent of $N_{\mathrm{H}}$. This is the result of the nonlinear limitation by the total amount of energy $E_{\mathrm{sn}}$. During the Sedov phase the shock speed $V_{\mathrm{s}}(t)$ depends only on $E_{\mathrm{sn}} / N_{\mathrm{H}}$, as we have seen before (see also Fig. 1a), whereas the Alfvénic shock Mach number $M_{\mathrm{a}}=V_{\mathrm{s}} / c_{\mathrm{a}} \propto \sqrt{N_{\mathrm{H}}}$ increases with $N_{\mathrm{H}}$, because the Alfvén speed $c_{\mathrm{a}} \propto 1 / \sqrt{N_{\mathrm{H}}}$. This leads to a stronger shock modification (see Fig. 2), that in turn provides a more efficient $\mathrm{CR}$ production $E_{\mathrm{c}} / E_{\mathrm{sn}}$ for larger $N_{\mathrm{H}}$. In spherical symmetry $E_{\mathrm{c}}$ can reach more than $50 \%$ of $E_{\mathrm{sn}}$ in this way. In reality, however, this spherical symmetry does not exist, because for a SN explosion in a uniform medium, as for SN 1006, the external magnetic field will also be uniform. This leads to a severe reduction of the injection rate around those parts of the shock surface that are roughly parallel to the external field and requires a renormalization of the overall acceleration efficiency by a factor of the order of 0.2 (Völk et al. 2003). This dipolar picture of the synchrotron emission has been confirmed in an elegant analysis of recent $X M M$ data for SN 1006 by Rothenflug et al. (2004). Altogether this leads to a rough proportionality $E_{\mathrm{c}} \propto N_{\mathrm{H}}$ until particles start to leave the remnant. As one can see from Fig. 6, the normalization of $E_{\mathrm{c}}$ by $E_{\mathrm{sn}}$ removes the main density dependence: the relative difference $E_{\mathrm{c}}\left(N_{\mathrm{H}}=0.3\right) / E_{\mathrm{c}}\left(N_{\mathrm{H}}=0.05\right)-1$ of the $E_{\mathrm{c}}$ values



Fig. 7. Integral $\pi^{0}$-decay (thick lines) and IC (thin lines) $\gamma$-ray fluxes from the NE half of the remnant as a function of $\gamma$-ray energy for $B_{0}=30 \mu \mathrm{G}$, and for the same different densities as in Fig. 1. The CANGAROO-I (open circles with error bars) and CANGAROO-II (solid circle with error bars) flux from the NE rim (Hara 2002) and the corresponding HESS upper limit for the CANGAROO position (Aharonian et al. 2005) are shown as well.

equals about 11 , whereas the relative difference of the normalized values $E_{\mathrm{c}} / E_{\mathrm{sn}}$ is about unity.

\section{Gamma-ray fluxes}

The previous considerations determine the values of the proton injection rate, the electron/proton ratio and the magnetic field strength $B_{0}$. The only important parameter which cannot be determined from the analysis of the synchrotron emission data is the external density $N_{\mathrm{H}}$. Therefore we now compare calculations for different $N_{\mathrm{H}}$ with the existing $\gamma$-ray measurements.

Figures 7 and 8 show the calculated integral $\gamma$-ray fluxes from the North-Eastern (NE) half of the remnant, in order to compare with the reported CANGAROO emission from the NE rim and the corresponding upper limit from HESS. These calculated fluxes correspond to 50 percent of the entire theoretical $\gamma$-ray flux from SN 1006 under our assumption of dipolar symmetry (Völk et al. 2003).

The key point is that a decrease of the ISM density leads to a considerable reduction of the expected $\pi^{0}$-decay $\gamma$-rays (see Fig. 7): in the Sedov phase the $\pi^{0}$-decay $\gamma$-ray flux $F_{\gamma}^{\pi} \propto N_{\mathrm{H}} E_{\mathrm{c}}$ is proportional to the gas density and to the CR energy content $E_{\mathrm{c}}$. This roughly implies $F_{\gamma}^{\pi} \propto N_{\mathrm{H}}^{2}$, as shown in the previous section and in agreement with the numerical results. In addition, an increase of the gas density leads to an increase of the Alfvénic shock Mach number and of the shock modification due to CR backreaction. This implies in turn a harder CR spectrum and correspondingly an increase of the $\gamma$-ray flux at the highest energies. Only this last factor influences the IC $\gamma$-ray flux. Therefore it is only weakly sensitive to the gas density as Fig. 7 also indicates.

To allow for a simple reading of the figures, we present the same data in two separate figures, Figs. 7 and 8a, showing in 


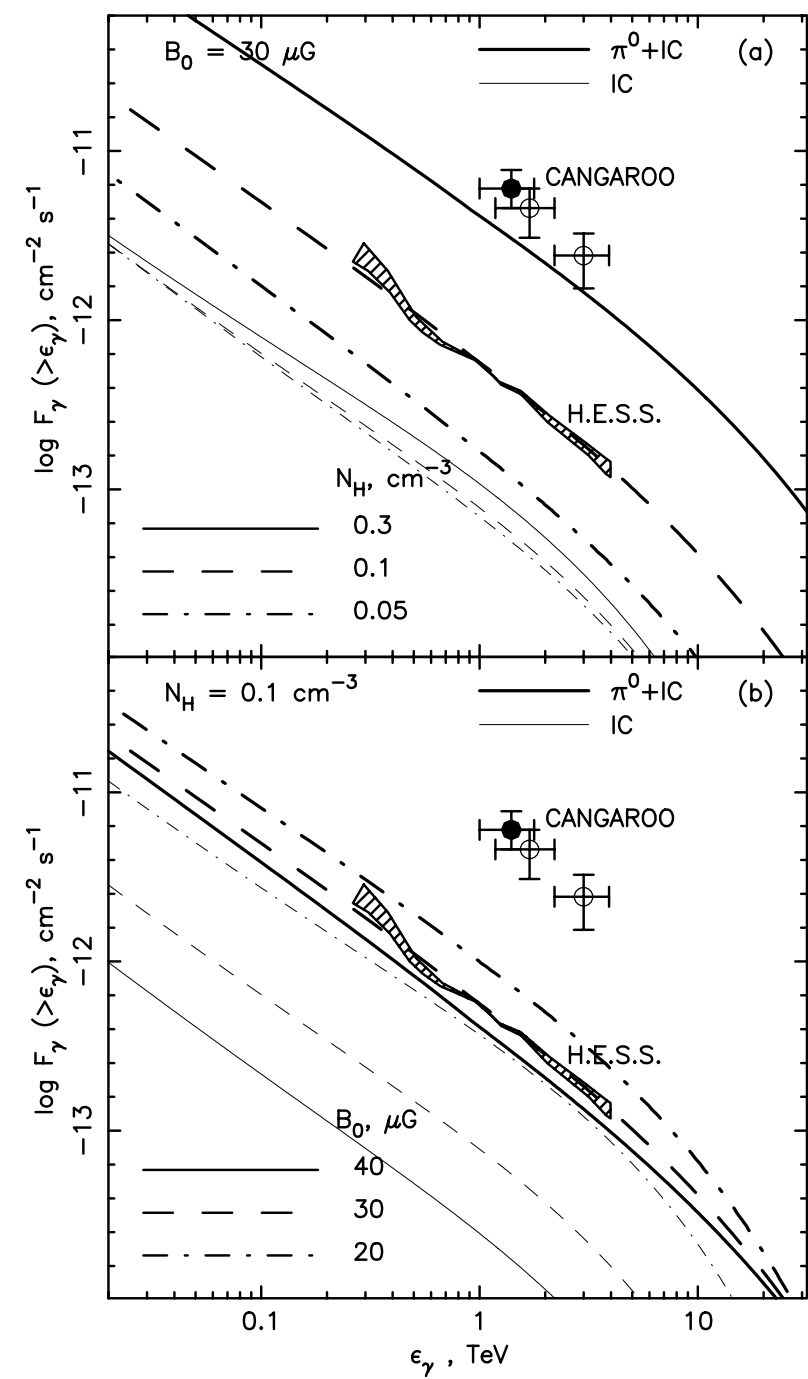

Fig. 8. Total $\left(\pi^{0}\right.$-decay + IC) (thick lines) and IC (thin lines) integral $\gamma$-ray fluxes from the NE half of the remnant as a function of $\gamma$-ray energy for the same cases as in Fig. 1. The CANGAROO-I (open circles with error bars) and CANGAROO-II (solid circle with error bars) flux from the NE rim (Hara 2002) and the corresponding HESS upper limit for the CANGAROO position (Aharonian et al. 2005) are shown as well.

Fig. 7 the theoretical $\pi^{0}$-decay $F_{\gamma}^{\pi}$ and IC $F_{\gamma}^{\mathrm{IC}}$ integral fluxes, whereas in Fig. 8a we show total fluxes $F_{\gamma}=F_{\gamma}^{\pi}+F_{\gamma}^{\mathrm{IC}}$ and $F_{\gamma}^{\mathrm{IC}}$.

In Fig. 8 we show the total $\left(\pi^{0}\right.$-decay + IC) and the IC integral $\gamma$-ray fluxes, again from the NE half of the remnant as a function of $\gamma$-ray energy for the same cases as in Fig. 1. Also the total $\gamma$-ray flux varies strongly with the ISM density (Fig. 7).

A modification of the magnetic field strength would not change much the $\pi^{0}$-decay $\gamma$-ray flux, and thus the total $\gamma$-ray flux, whereas the IC flux decreases considerably with increasing magnetic field strength. Figure $8 \mathrm{~b}$ illustrates this fact, even though we consider the value $B_{0} \approx 30 \mu \mathrm{G}$ as experimentally quite well determined.

Note that an Inverse Compton $\gamma$-ray emission scenario in a low magnetic field of order $B_{\mathrm{d}}=4 \pm 1 \mu \mathrm{G}$ (Tanimori et al. 2001) is clearly excluded by the HESS upper limits, and this is fully consistent with the measured and expected field amplification.

The non-detection of SN 1006 by the HESS experiment (Aharonian et al. 2005) implies that the hydrogen density $N_{\mathrm{H}}$ is lower than $0.1 \mathrm{~cm}^{-3}$, and excludes the high value $N_{\mathrm{H}}=$ $0.3 \mathrm{~cm}^{-3}$ which happened to fit the CANGAROO data. As clearly shown in Fig. 8a, at $1 \mathrm{TeV}$ and for $B_{0}=30 \mu \mathrm{G}$ the HESS upper limit is still about three times larger than the total $\gamma$-ray flux from SN 1006, expected for $N_{\mathrm{H}}=0.05 \mathrm{~cm}^{-3}$, which is at the lower end of the range $0.05 \leq N_{\mathrm{H}} \leq 0.3 \mathrm{~cm}^{-3}$ of plausible ambient densities.

Since the IC $\gamma$-ray emission is quite insensitive to the ISM density, the lower limit for the expected $\gamma$-ray flux at TeV energies - as derived from the integrated synchrotron flux and the field amplification alone - is about a factor of five lower than the HESS upper limit. Such a low $\gamma$-ray flux from SN 1006 would be expected if the ambient ISM number density $N_{\mathrm{H}}$ was even considerably lower than $0.05 \mathrm{~cm}^{-3}$.

\section{Summary}

Our reexamination of the most relevant set of physical and astronomical parameters of SN 1006 led us to chose the somewhat greater source distance of $2.2 \mathrm{kpc}$, determined by more recent measurements, and to establish the interior magnetic field in SN 1006 more precisely at $B_{\mathrm{d}} \approx 150 \mu \mathrm{G}$. Most important, however, is another fact: the lack of a TeV signal from SN 1006 that follows from the non-detection by the HESS instrument does not invalidate the theoretical picture which gives a consistent description of the nonthermal emission characteristics; it rather implies a constraint on the ambient gas density $N_{\mathrm{H}}<0.1 \mathrm{~cm}^{-3}$. This point was discussed in detail. The renormalization of the $\gamma$-ray flux on which this result is partly based has been recently confirmed experimentally. The well-defined morphology makes this remnant the simplest and best understood of its kind. In conjunction with the amplification of the magnetic field by the accelerated cosmic rays, the HESS nondetection completely rules out the low-field Inverse Compton scenarios that were popular until a few years ago.

In the case of SN 1006 and in other similar cases $\gamma$-ray measurements will at the same time give a reliable estimate of the ambient ISM density.

Quantitatively (see Figs. 7 and 8a), the present HESS upper limit is only a factor of about three above the total TeV flux expected for $N_{\mathrm{H}}=0.05 \mathrm{~cm}^{-3}$, which is at the lower end of the range $0.05 \leq N_{\mathrm{H}} \leq 0.3 \mathrm{~cm}^{-3}$ discussed in the literature. If the actual ISM is so diluted that $N_{\mathrm{H}}<0.05 \mathrm{~cm}^{-3}$, then the lowest expected TeV $\gamma$-ray flux is by a factor of five below the present HESS upper limit.

Given the arguments above, it is certainly worth attempting the detection of this important source in a deep observation of about $200 \mathrm{~h}$ with the HESS experiment.

Acknowledgements. We thank the referee for a number of valuable suggestions to improve the paper. This work has been supported in part by the Russian Foundation for Basic Research (grant 03-02-16524) and LSS 422.2003.2. 


\section{References}

Aharonian, F. A., Akhperjanian, A. G., Aye, K.-M. H., et al. 2005 [arXiv: astro-ph/0502239]

Allen, G. E., Gotthelf, E. V., \& Petre, R. 1999, Proc. 26th ICRC, Salt Lake City, 3, 480

Bamba, A., Yamazaki, R., Ueno, M., \& Koyama, K. 2003, ApJ, 589, 827

Bell, A. R. 2004, MNRAS, 353, 550

Bell, A. R., \& Lucek, S. G. 2001, MNRAS, 321, 433

Berezhko, E. G., \& Völk, H. J. 1997, Astroparticle Phys., 7, 183

Berezhko, E. G., \& Ellison, D. C. 1999, ApJ, 526, 385

Berezhko, E. G., \& Völk, H. J. 2004, A\&A, 419, L27

Berezhko, E. G., Elshin, V. K., \& Ksenofontov, L. T. 1996, J. Exp. Theor. Phys., 82, 1

Berezhko, E. G., Ksenofontov, L. T., \& Völk, H. J. 2002, A\&A, 395, 943

Berezhko, E. G., Pühlhofer, G., \& Völk, H. J. 2003a, A\&A, 400, 971

Berezhko, E. G., Ksenofontov, L. T., \& Völk, H. J. 2003b, A\&A, 412, L11

Chevalier, R. A. 1982, ApJ, 258, 790
Dubner, G. M., Giacani, E. B., Goss, W. M., et al. 2002, A\&A, 387, 1047

Dwarkadas, V. V., \& Chevalier, R. A. 1998, ApJ, 497, 807

Hamilton, A. J. S., Sarazin, C. L., \& Szymkowiak, A. E. 1986, ApJ, 300,698

Hara, S. 2002, Doctoral Thesis, Tokyo Institute of Technology Jones, E. M., Smith, B. W., \& Straka, W. C. 1981, ApJ, 249, 185

Koyama, K., Petre, R., Gotthelf, E. V., et al. 1995, Nature, 378, 255

Lucek, S. G., \& Bell, A. R. 2000, MNRAS, 314, 65

Moffett, D. A., Goss, W. M., \& Reynolds, S. P. 1993, AJ, 106, 1566

Reynolds, S. P. 1996, ApJ, 459, L13

Rothenflug, R., Ballet, J., Dubner, G., et al. 2004, A\&A, 425, 121

Tanimori, T., Hayami, Y., Kamei, S., et al. 1998, ApJ, 497, L25

Tanimori, T., Naito, T., Yoshida, T., et al. 2001, Proc. 27th ICRC, Hamburg, 6, 2465

Völk, H. J. 2004, Proc. 28th ICRC, Tsukuba, Invited, Rapporteur, and Highlight Papers, 8, 29

Völk, H. J., Berezhko, E. G., \& Ksenofontov, L. T. 2003, A\&A, 409, 563

Völk, H. J., Berezhko, E. G., \& Ksenofontov, L. T. 2005, A\&A, 433, 229

Winkler, P. F., Gupta, G., \& Long, K. S. 2003, ApJ, 585, 324 